Article

\title{
Palladium-Catalyzed Amination of Dichloroquinolines with Adamantane-Containing Amines
}

Anton S. Abel ${ }^{1}$, Alexei D. Averin ${ }^{1, *}$, Olga A. Maloshitskaya ${ }^{1}$, Evgenii N. Savelyev ${ }^{2}$,

Boris S. Orlinson ${ }^{2}$, Ivan A. Novakov ${ }^{2}$ and Irina P. Beletskaya ${ }^{1}$

1 Department of Chemistry, Lomonosov Moscow State University, Leninskie Gory, 1-3, Moscow 119991, Russia; E-Mails: antonabel@list.ru (A.S.A.); maloshitskaya@mail.ru (O.A.M.); beletska@org.chem.msu.ru (I.P.B.)

2 Volgograd State Technical University, 28 Lenina prosp., Volgograd 400131, Russia;

E-Mails: phanchem@vstu.ru (E.N.S.; B.S.O.); rector@vstu.ru (I.A.N)

* Author to whom correspondence should be addressed; E-Mail: alexaveron@yandex.ru;

Tel.: +7-495-939-1139; Fax: +7-495-939-3618.

Received: 31 December 2012; in revised form: 24 January 2013 / Accepted: 1 February 2013 /

Published: 6 February 2013

\begin{abstract}
Pd-catalyzed amination of isomeric 2,6-, 2,8-, 4,8- and 4,7-dichloroquinolines was studied using adamantane-containing amines in which substituents at the nitrogen atom differ in bulkiness. The selectivity of the amination of 2,6-dichloroquinoline was very low, substantially better results were obtained with 2,8-dichloroquinoline, and 4,8- and 4,7-dichloroquinolines provided the best yields of the amination products. Diamination of 4,8- and 4,7-dichloroquinolines was carried out with two amines which differ strongly in the bulkiness of the alkyl group. In the majority of cases BINAP ligand was successfully applied, however, it had to be replaced with DavePhos in certain reactions when using the most sterically hindered amine as well as for the diamination reactions.
\end{abstract}

Keywords: amines; adamantane; Pd catalysis; amination; quinoline

\section{Introduction}

The pharmacological activity of adamantane derivatives is well documented and arises from various factors, the main being their ability to penetrate the lipid layers of membranes and to interact with hydrophobic sites of proteins due to the presence of a rigid lipophilic backbone. A special place among 
these compounds is occupied by the amines with adamantane-containing substituents which have already found medical applications, like adamantan-1-amine (amantadine) [1], (1-adamantylmethyl)amine hydrochloride (rimantadine) [2], 1,3-dimethyladamantan-5-amine (memantine) [3]. Biological studies of the adamantane derivatives bearing heterocyclic substituents, e.g., pyridine, pyrazole, benzimidazole, and other $N$-heterocycles are reported [4], and their psychotropic activity was especially addressed. However, there is scarce information about the quinolinyl derivatives of adamantaneamines, as only two publications [5,6] deal with 4-quinolinyl derivatives which demonstrated anti-malarial activity, and one describes the synthesis of 2-quinolinyl-substituted adamantane-1,3-diamine [7]. All these compounds were obtained using non-catalytic methods.

In general, the successful application of catalytic amination for the synthesis of aminoquinoline compounds from corresponding halogen derivatives depends strongly on the position of the halogen atom in the quinoline moiety. Pd-catalyzed amination reactions [8-13] are the most frequently used for this purpose. There are enough examples of the Pd-catalyzed amination of 2-chloroquinoline [14-18], 3-bromo- and 3-chloroquinolines [13-23], 6-bromoquinoline [24,25]. Less studied are the Pd-catalyzed aminations of 5-bromoquinoline [26,27], 8-chloro- and 8-bromoquinolines [14,28]. The Cu-catalyzed amination of 3-haloquinolines [29-31] and of 5-bromoquinoline [32] has also been reported, while there is almost no information about the application of the catalytic approaches for the synthesis of 4-aminoquinoline derivatives except for one work [33], although 4-amino- and especially 4-amino-7chloroquinolines were shown to be potent anti-malarial agents [34-36].

Taking all these facts into consideration and in view of our interest in the Buchwald-Hartwig amination of heteroaryl halides [37-40], we decided to study the Pd-catalyzed amination reactions of isomeric dichloroquinolines with selected adamantane-containing amines, which differ in the steric hindrance at the amino group, and to determine the best conditions for the selective mono- and diamination of these hetaryl dichlorides.

\section{Results and Discussion}

We investigated the Pd-catalyzed amination of commercially available 2,6-, 2,8-, 4,8- and 4,7-dichloroquinolines which possess two chlorine atoms of different reactivity. The four adamantanecontaining amines 1a-d studied in the amination reactions have different substituents at the nitrogen atom. Catalytic reactions were carried out using $\mathrm{Pd}(\mathrm{dba})_{2}$ as a source of $\operatorname{Pd}(0)$, phosphine ligands BINAP (2,2'-bis(diphenylphosphino)-1,1'-binaphthalene) or DavePhos (2-dicyclohexylphosphino-2'dimethylaminobiphenyl), and sodium tert-butoxide as a base. The reactions were conducted in boiling dioxane $(\mathrm{c}=0.1 \mathrm{M})$, equimolar amounts of reagents were employed for the synthesis of monoaminosubstituted quinolines, 3-4 equiv. of amine were used to obtain diamino-substituted products. All four amines were studied in the monoamination reactions with each dichloroquinoline, and two amines, namely 1a and 1d, were employed in the diamination processes. Generally, the reactions were run for $6-8 \mathrm{~h}$ in the case of monoamination processes and $15 \mathrm{~h}$ in the case of diamination processes to achieve full consumption of the starting materials. The reaction products were isolated by column chromatography on silica gel. First, we carried out the reactions of 2,6-dichloroquinoline which was thought to be suitable for the synthesis of monoaminated products due to the presence of a reactive chlorine atom in position 2 and a much less reactive chlorine in 
position 6. However, all our attempts to obtain the monoamino or diamino derivative failed. The reactions with 1 or 4 equiv. of the amine 1a catalyzed with 4 or $8 \mathrm{~mol} \%$ of the catalyst, in the presence of BINAP or DavePhos, provided only complicated reaction mixtures which could not be separated by the chromatography. Such behavior of 2,6-dichloroquinoline may be explained by a high reactivity of the chlorine atom at position 2 which promotes the $N$-arylation and $N, N$-diarylation reactions as well as other substitution processes like alkoxylation with $t$-BuONa and homocoupling. Moreover, the substitution of the chlorine atom at position 2 markedly affects the substitution of the chlorine atom at position 6 due to a mesomeric effect. Thus we gave up attempts to work with 2,6-dichloroquinoline and turned to its isomer, 2,8-dichloroquinoline (Scheme 1).

Scheme 1. Pd-catalyzed amination of 2,8-dichloroquinoline.

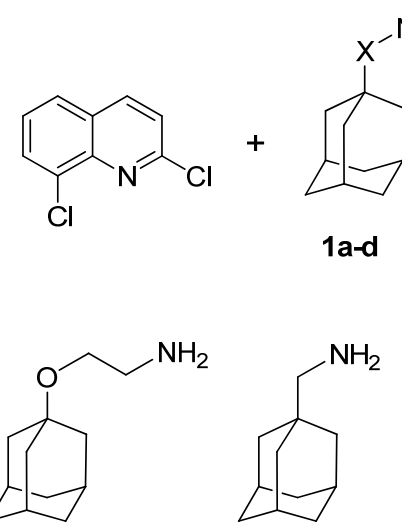

$1 \mathrm{a}$

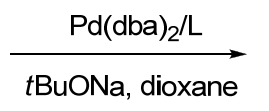

$1 \mathrm{~b}$

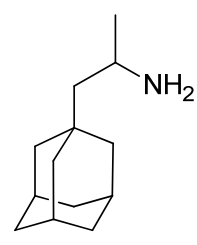

$1 c$

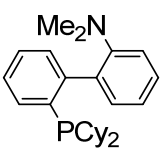

DavePhos
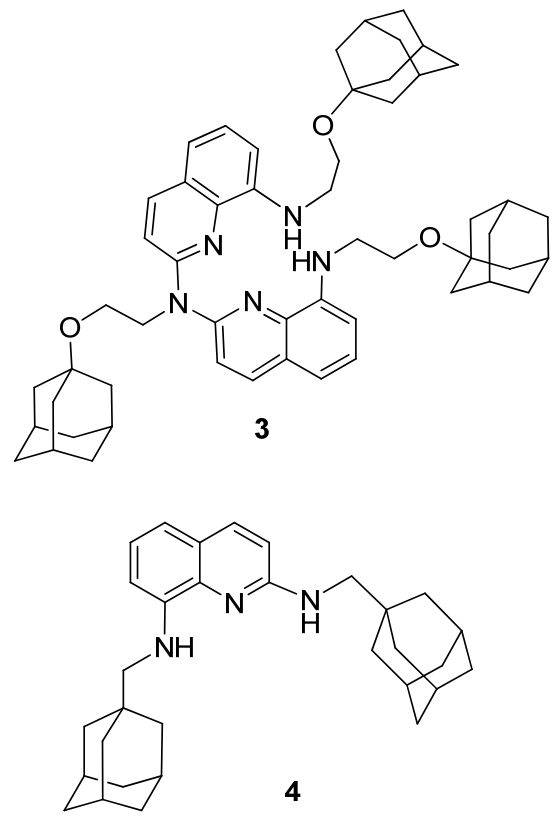

This compound also possesses two chlorine atoms with distinctively different reactivity. The reaction with a less sterically hindered amine 1a catalyzed by 4 mol\% catalyst with BINAP as ligand provided $64 \%$ yield of the monoamination product $\mathbf{2 a}$ in which a more reactive chlorine atom was substituted for the amino group (Table 1, entry 1). The attempt to obtain the diamination product by reacting 2,8-dichloroquinoline with 4 equiv. of the amine 1a in the presence of the twofold amount of the same catalyst gave rise to an inseparable mixture (entry 2), and the diamination product could not be isolated as an individual product. The change of BINAP for the electron-donor DavePhos led to the formation of one main product 3 , which is the result of the combination of the diamination and $\mathrm{N}, \mathrm{N}$-diarylation processes (entry 3 ). It was isolated in $41 \%$ yield, and no desired diamino compound was obtained. The ${ }^{1} \mathrm{H}-\mathrm{NMR}$ spectrum of compound $\mathbf{3}$ is characterized by two distinctively different $\mathrm{NCH}_{2} \mathrm{CH}_{2} \mathrm{O}$ fragments. The first, bearing two quinolinyl substitutents at the nitrogen atom, possesses downfield-shifted $\mathrm{CH}_{2} \mathrm{~N}$ protons $\left(\delta 4.58 \mathrm{ppm}\right.$ ), while in the second fragment $\mathrm{CH}_{2} \mathrm{~N}$ protons are observed at $3.4 \mathrm{ppm}$. In the ${ }^{13} \mathrm{C}$-NMR spectrum the corresponding carbon atoms are observed at 49.8 and 43.9 ppm, respectively. To compare, in the compound $\mathbf{2 a}$ the protons of the $\mathrm{CH}_{2} \mathrm{~N}$ group are observed at $3.7 \mathrm{ppm}$ and corresponding carbon atom possesses the chemical shift $42.0 \mathrm{ppm}$. The 
downfield shift of both protons and carbon atoms in $\mathrm{CH}_{2} \mathrm{NQuin}_{2}$ fragment compared to $\mathrm{CH}_{2} \mathrm{NHQuin}$ is very characteristic [40] and verifies the structure of the compound 3. Additional support is provided by a pronounced downfield shift of the H3, H3' protons of the quinolinyl moieties in compound 3 (7.47 ppm compared to $6.67 \mathrm{ppm}$ in $N$-monoaryl derivative 2a).

Table 1. Pd-catalyzed amination of 2,8-dichloroquinoline.

\begin{tabular}{cccccc}
\hline Entry & Amine & Equiv. of amine & Ligand & Pd(dba) $\mathbf{2} / \mathbf{L}, \mathbf{m o l} \%$ & Product, yield \\
\hline 1 & 1a & 1 & BINAP & $4 / 4.5$ & 2a, 64\% \\
2 & 1a & 4 & BINAP & $8 / 9$ & inseparable mixture \\
3 & 1a & 4 & DavePhos & $8 / 9$ & $\mathbf{3}, 41 \%$ \\
4 & 1b & 1 & BINAP & $4 / 4.5$ & $\mathbf{2 b}, 42 \% ; \mathbf{4}, 26 \%$ \\
5 & 1c & 1 & BINAP & $4 / 4.5$ & $\mathbf{2 c}, 56 \%$ \\
6 & 1d & 1 & BINAP & $4 / 4.5$ & no amination \\
7 & 1d & 3 & BINAP & $8 / 9$ & inseparable mixture \\
8 & 1d & 1 & DavePhos & $4 / 4.5$ & 2d, $42 \%$ \\
9 & 1d & 3 & DavePhos & $8 / 9$ & inseparable mixture \\
\hline
\end{tabular}

The reaction with one equivalent of a more sterically hindered amine $\mathbf{1 b}$ unexpectedly gave not only the target monoamino derivative $\mathbf{2 b}$, but also some amount of the diamination product $\mathbf{4}$ (entry 4 ). This fact can be explained by the impossibility of $N, N$-diarylation in this case due to steric hindrances in the amine 1b. Amine 1c, in which the amino group bears a substituent with a tertiary carbon atom, is somewhat less active and provided 56\% yield of the monoamination product $2 \mathrm{c}$ (entry 5). It was necessary to increase the reaction time to $15 \mathrm{~h}$ to ensure full consumption of the starting materials. The reaction with the most sterically hindered amine 1d could not be catalyzed with 4 mol\% catalyst using BINAP (entry 6), but the application of DavePhos solved the problem, and the target compound 2d was obtained in $42 \%$ yield (entry 8 ). It was found impossible to synthesize diaminosubstituted quinoline using the excess of amine and twofold amount of the catalyst either in the presence of BINAP or DavePhos ligands (entries 7 and 9). In all runs the reaction mixtures contained numerous compounds which could not be separated by the column chromatography. In many cases the formation of 2-tert-butoxy-substituted quinolines was noted, due to the non-catalytic substitution of the chlorine atom, and this process diminished the yields of the target amination products. The most upfield-shifted proton of the quinoline moiety in the compounds of type $2(\delta 6.6-6.7 \mathrm{ppm})$ possesses the largest coupling constant $\left({ }^{3} J=8.8 \mathrm{~Hz}\right)$ with the most downfield-shifted proton $(\delta 7.7-7.8 \mathrm{ppm})$. This fact unambiguously supports the structure of these compounds with the amino group in position 2 of the quinoline system because ${ }^{3} J_{\mathrm{H} 3 \mathrm{H} 4}$ is the largest coupling constant in quinolines and the difference in chemical shifts of these protons in 2-aminoquinolines is the biggest.

If 2,8-dichloroquinoline was found to be quite capricious in the Pd-catalyzed amination, its isomer, 4,8-dichloroquinoline, proved to react in a smoother manner (Scheme 2). The reaction of the most active amine 1a under the convenient catalytic conditions provided $77 \%$ yield of the product of the compound 5a (Table 2, entry 1). It was impossible to obtain the 4,8-diaminosubstituted quinoline 6a with 4 equiv. of this amine using BINAP as a ligand (entry 2), however, the application of DavePhos promoted this reaction (entry 3). The monoamination processes ran normally for all other amines $\mathbf{1 b}-\mathbf{d}$ and the yields of the 4-amino-8-chloroquinolines ranged from 67 to $84 \%$ (entries $4-6$ ). These better 
results compared to the reactions with 2,8-dichloroquinoline can be attributed to a lower reactivity of the chlorine atom in the position 4 due to steric reasons and to the absence of the possible side reactions like $N, N$-diarylation. These results are in a good correspondence with our recent observations of the catalytic amination of 2- and 4-chloroquinolines [40]. The diamination of 4,8-dichloroquinoline with the most hindered amine 1d is possible when using DavePhos ligand (entry 8), whereas in the presence of BINAP the reaction gave only the monoamination product $\mathbf{5 d}$ almost in the same yield as it was with one equivalent of amine 1d (entry 7). The most upfield-shifted proton of the quinoline moiety in the compounds of type $5(\delta 6.2-6.5 \mathrm{ppm})$ possesses the smallest coupling constant $\left({ }^{3} \mathrm{~J}=5.4 \mathrm{~Hz}\right)$ with the most downfield-shifted proton $(\delta 8.4-8.6 \mathrm{ppm})$. This fact unambiguously supports the structure of these compounds with the amino group in the position 4 of the quinoline system because ${ }^{3} J_{\mathrm{H} 2 \mathrm{H} 3}$ is the smallest coupling constant in quinolines and the difference in chemical shifts of these protons in 4-aminoquinolines is the biggest. The same observation is true for the compounds of type 7 (vide infra).

Scheme 2. Pd-catalyzed amination of 4,8-dichloroquinoline.<smiles>Clc1ccnc2c(Cl)cccc12</smiles><smiles>[131I-]</smiles>
1a-d
$\operatorname{Pd}(\mathrm{dba})_{2} / \mathrm{L}$ tBuONa, dioxane<smiles>[X]C12CC3CC(CC(C3)C1)C2</smiles>

5a-d<smiles>[X]C(C)(CC)Nc1cccc2c(NC34CC5CC(CC(C5)C3)C4)ccnc12</smiles>

$6 a, d$

Table 2. Pd-catalyzed amination of 4,8-dichloroquinoline.

\begin{tabular}{cccccc}
\hline Entry & Amine & Equiv. of amine & Ligand & Pd(dba) $)_{2} / \mathbf{L}, \mathbf{m o l} \%$ & Product, yield \\
\hline 1 & 1a & 1 & BINAP & $4 / 4.5$ & $\mathbf{5 a}, 77 \%$ \\
2 & 1a & 4 & BINAP & $8 / 9$ & inseparable mixture \\
3 & 1a & 4 & DavePhos & $8 / 9$ & $\mathbf{6 a}, 52 \%{ }^{\text {a }}$ \\
4 & 1b & 1 & BINAP & $4 / 4.5$ & $\mathbf{5 b}, 67 \%$ \\
5 & 1c & 1 & BINAP & $4 / 4.5$ & $\mathbf{5 c}, 84 \%$ \\
6 & 1d & 1 & BINAP & $4 / 4.5$ & $\mathbf{5 d}, 70 \%$ \\
7 & 1d & 3 & BINAP & $8 / 9$ & $\mathbf{5 d}, 68 \%$ \\
8 & 1d & 3 & DavePhos & $8 / 9$ & $\mathbf{6 d}, 45 \%$ \\
\hline
\end{tabular}

${ }^{\mathrm{a}}$ Contains admixtures.

As it has been already mentioned, the halogen atom in the position 7 of the quinoline ring is much less active than those in the positions 2 and 4, what should result in a better selectivity of the monoamination process. The reactions of 4,7-dichloroquinoline supported this idea (Scheme 3). The reaction with the amine 1a afforded 52\% yield of the 4-amino-7-chloroquinoline 7a (Table 3, entry 1), while the diamination process was successful with either BINAP or DavePhos ligands (entries 2 and 3) providing almost equal amounts of the diaminated product 8a. The reactions with the amines $\mathbf{1 b}-\mathbf{d}$ were successful as well, giving corresponding monoaminated products $7 \mathbf{b}-\mathbf{d}$ in $61-79 \%$ yields (entries $4-6$ ). 
Diamination in the presence of $\mathrm{Pd}(\mathrm{dba})_{2} / \mathrm{BINAP}$ system ran normally, even with the most bulky amine 1d, affording diamino derivative $\mathbf{8 d}$ in 58\% yield (entry 7). Diamino-substituted quinolines $6 \mathbf{d}$ and $\mathbf{8 d}$ are formed as pairs of diastereomers due to the presence of an asymmetric carbon atom in the structure of parent amine 1d. Nevertheless, their ${ }^{1} \mathrm{H}$ and ${ }^{13} \mathrm{C}$ spectra show almost no splitting of the signals due to a long distance between the substituents bearing asymmetric carbon atom, their free rotation and the presence of a planar heteroaromatic spacer.

Scheme 3. Pd-catalyzed amination of 4,7-dichloroquinoline.

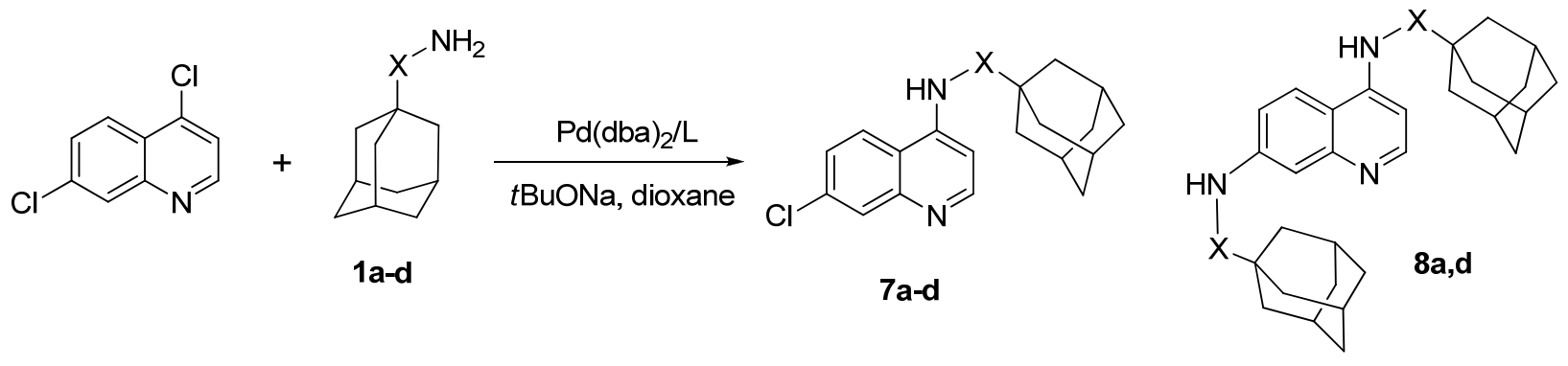

Table 3. Pd-catalyzed amination of 4,7-dichloroquinoline.

\begin{tabular}{cccccc}
\hline Entry & Amine & Equiv. of amine & Ligand & Pd(dba) $\mathbf{~}_{\mathbf{2}} / \mathbf{L}, \mathbf{m o l} \%$ & Product, yield \\
\hline 1 & 1a & 1 & BINAP & $4 / 4.5$ & $\mathbf{7 a}, 52 \%$ \\
2 & 1a & 4 & BINAP & $8 / 9$ & $\mathbf{8 a}, 67 \%$ \\
3 & 1a & 4 & DavePhos & $8 / 9$ & $\mathbf{8 a}, 71 \%$ \\
4 & 1b & 1 & BINAP & $4 / 4.5$ & $\mathbf{7 b}, 61 \%$ \\
5 & 1c & 1 & BINAP & $4 / 4.5$ & $\mathbf{7 c}, 79 \%$ \\
6 & 1d & 1 & BINAP & $4 / 4.5$ & $\mathbf{7 d}, 77 \%$ \\
7 & 1d & 3 & BINAP & $8 / 9$ & $\mathbf{8 d}, 58 \%$ \\
\hline
\end{tabular}

\section{Experimental}

\section{General}

NMR spectra were registered using a Bruker Avance 400 spectrometer, MALDI-TOF spectra were obtained with a Bruker Autoflex II spectrometer using 1,8,9-trihydroxyanthracene as a matrix and PEGs as internal standards. Isomeric dichloroquinolines, BINAP and DavePhos ligands, sodium tert-butoxide were purchased from Aldrich and Acros and used without further purification. Amine 1a was obtained according to a reported procedure [41], as was amine 1b [42], while amines 1c,d were obtained according to a method described in ref. [43]. $\mathrm{Pd}(\mathrm{dba})_{2}$ was synthesized from $\mathrm{PdCl}_{2}$ according to a known procedure [44]. Dioxane was distilled over $\mathrm{NaOH}$ followed by the distillation over sodium under argon. Acetonitrile, dichloromethane and methanol were used freshly distilled.

\section{Palladium-Catalyzed Amination of Dichloroquinolines-General Method}

A two-neck flask equipped with a condenser and a magnetic stirrer, flushed with dry argon, was charged with corresponding dichloroquinoline (50 mg, $0.25 \mathrm{mmol}), \mathrm{Pd}(\mathrm{dba})_{2}(6-12 \mathrm{mg}, 4-8 \mathrm{~mol} \%$ ), BINAP or DavePhos ligand (4.5-9 mol\%), and absolute dioxane (2 mL). The mixture was stirred for 
2-3 min, then corresponding amine 1a-d $(0.25$ or $0.75-1 \mathrm{mmol})$ and $t \mathrm{BuONa}(0.375 \mathrm{mmol}$ or $0.75 \mathrm{mmol})$ were added, and the reaction mixture was refluxed for 6-15 h. After cooling it down to ambient temperature the reaction mixture was diluted with $\mathrm{CH}_{2} \mathrm{Cl}_{2}$, the solution filtered and evaporated in vacuo, and the residue was chromatographed on silica gel using a sequence of eluents: petrol ether, petrol ether- $\mathrm{CH}_{2} \mathrm{Cl}_{2}$ 2:1-1:1, $\mathrm{CH}_{2} \mathrm{Cl}_{2}, \mathrm{CH}_{2} \mathrm{Cl}_{2} / \mathrm{MeOH}$ 200:1-3:1.

N-[2-(1-Adamantyloxy)ethyl]-8-chloroquinolin-2-amine (2a). Obtained from 2,8-dichloroquinoline $(50 \mathrm{mg})$, amine 1a (49 mg) in the presence of Pd(dba) 2 (6 mg), BINAP (7 mg) and $t$-BuONa (36 mg). Eluent $\mathrm{CH}_{2} \mathrm{Cl}_{2} / \mathrm{MeOH}$ 100:1. Yield $57 \mathrm{mg}$ (64\%), yellowish viscous oil. ${ }^{1} \mathrm{H}-\mathrm{NMR}\left(\mathrm{CDCl}_{3}\right) \delta 1.55-1.68$ (m, 6H), 1.76 (br.s, 6H), 2.14 (br.s, 3H), 3.66-3.70 (m, 2H), 3.70-3.75 (m, 2H), 5.27 (br.s, 1H), 6.67 $(\mathrm{d}, J=8.7 \mathrm{~Hz}, 1 \mathrm{H}), 7.08\left(\mathrm{dd}, J_{o b s}=7.7,7.7 \mathrm{~Hz}, 1 \mathrm{H}\right), 7.47(\mathrm{~d}, J=7.8 \mathrm{~Hz}, 1 \mathrm{H}), 7.62(\mathrm{~d}, J=7.5 \mathrm{~Hz}, 1 \mathrm{H})$, $7.76(\mathrm{~d}, J=8.7 \mathrm{~Hz}, 1 \mathrm{H}) .{ }^{13} \mathrm{C}-\mathrm{NMR}\left(\mathrm{CDCl}_{3}\right) \delta 30.5(3 \mathrm{C}), 36.4(3 \mathrm{C}), 41.6(3 \mathrm{C}), 42.0(1 \mathrm{C}), 58.7(1 \mathrm{C})$, 72.4 (1C), 112.6 (1C), 121.5 (1C), 124.5 (1C), 126.4 (1C), 129.5 (1C), 129.9 (1C), 137.3 (1C), 157.1 (1C), one quaternary carbon atom was not assigned due to line broadening. HRMS (MALDI-TOF): $\mathrm{C}_{21} \mathrm{H}_{26} \mathrm{ClN}_{2} \mathrm{O}(\mathrm{M}+\mathrm{H})^{+}$calcd; 357.1734 observed; 357.1769 .

$N^{2}, N^{8}$-bis [2-(1-Adamantyloxy)ethyl]- $N^{2}-(8-\{[2-(1-$ adamantyloxy)ethyl] amino $\}$ quinolin-2-yl)quinoline2,8-diamine (3). Obtained from 2,8-dichloroquinoline (50 mg), amine 1a (195 mg) in the presence of $\mathrm{Pd}(\mathrm{dba})_{2}(12 \mathrm{mg})$, DavePhos $(9 \mathrm{mg})$ and $t$-BuONa $(60 \mathrm{mg})$. Eluent $\mathrm{CH}_{2} \mathrm{Cl}_{2} / \mathrm{MeOH} 200: 1-100: 1$. Yield $57 \mathrm{mg}(64 \%)$, yellowish viscous oil. ${ }^{1} \mathrm{H}-\mathrm{NMR}\left(\mathrm{CDCl}_{3}\right) \delta 1.47-1.78(\mathrm{~m}, 36 \mathrm{H}), 2.06$ (br.s, 6H), 2.14 (br.s, 3H), 3.38-3.44 (m, 4H), 3.69 (t, $J=5.9 \mathrm{~Hz}, 4 \mathrm{H}), 3.84(\mathrm{t}, J=6.0 \mathrm{~Hz}, 2 \mathrm{H}), 4.58$ (t, $J=6.0 \mathrm{~Hz}$, 2H), 6.09 (br.s, 2H), 6.67 (d, $J=7.6 \mathrm{~Hz}, 1 \mathrm{H}), 6.96(\mathrm{~d}, J=7.3 \mathrm{~Hz}, 1 \mathrm{H}), 7.21$ (dd, $J_{o b s}=7.8,7.8 \mathrm{~Hz}$, 1H), $7.47(\mathrm{~d}, J=9.0 \mathrm{~Hz}, 1 \mathrm{H}), 7.87(\mathrm{~d}, J=9.0 \mathrm{~Hz}, 1 \mathrm{H}) .{ }^{13} \mathrm{C}-\mathrm{NMR}\left(\mathrm{CDCl}_{3}\right) \delta 30.4(9 \mathrm{C}), 36.4(9 \mathrm{C}), 41.5$ (9C), 43.9 (2C), 49.8 (1C), 58.0 (1C), 58.5 (2C), 72.2 (2C), 72.3 (1C), 105.4 (1C), 113.8 (1C), 116.4 (1C), 125.0 (1C), 125.5 (1C), 136.4 (1C), 137.0 (1C), 143.8 (1C), 153.9 (1C). HRMS (MALDI-TOF): $\mathrm{C}_{54} \mathrm{H}_{70} \mathrm{~N}_{5} \mathrm{O}_{3}(\mathrm{M}+\mathrm{H})^{+}$calcd.; 836.5479 observed; 836.5422 .

$N$-(1-Adamantylmethyl)-8-chloroquinolin-2-amine (2b). Obtained from 2,8-dichloroquinoline (50 mg), amine $\mathbf{1 b}(41 \mathrm{mg})$ in the presence of $\mathrm{Pd}(\mathrm{dba})_{2}(6 \mathrm{mg})$, BINAP $(7 \mathrm{mg})$ and $t$-BuONa $(36 \mathrm{mg})$. Eluent petroleum ether- $\mathrm{CH}_{2} \mathrm{Cl}_{2} 1: 1$. Yield $34 \mathrm{mg}(42 \%)$, yellowish viscous oil. ${ }^{1} \mathrm{H}-\mathrm{NMR}\left(\mathrm{CDCl}_{3}\right) \delta 1.58-1.62$ (m, 6H), 1.62-1.75 (m, 6H), 1.99 (br.s, 3H), 3.23 (d, $J=5.6 \mathrm{~Hz}, 2 \mathrm{H}), 5.03$ (br.s, 1H), 6.70 (d, $J=8.8 \mathrm{~Hz}$, $1 \mathrm{H}), 7.06\left(\mathrm{dd}, J_{o b s}=7.7,7.7 \mathrm{~Hz}, 1 \mathrm{H}\right), 7.46(\mathrm{~d}, J=8.0 \mathrm{~Hz}, 1 \mathrm{H}), 7.62(\mathrm{~d}, J=7.5 \mathrm{~Hz}, 1 \mathrm{H}), 7.78(\mathrm{~d}$, $J=8.8 \mathrm{~Hz}, 1 \mathrm{H}) .{ }^{13} \mathrm{C}-\mathrm{NMR}\left(\mathrm{CDCl}_{3}\right) \delta 28.3(3 \mathrm{C}), 34.2(1 \mathrm{C}), 37.0(3 \mathrm{C}), 40.5(3 \mathrm{C}), 53.4(1 \mathrm{C}), 111.5$ (1C), 121.3 (1C), 124.4 (1C), 126.3 (1C), 129.6 (2C), 137.5 (1C), 143.5 (1C), 158.0 (1C). HRMS (MALDI-TOF): $\mathrm{C}_{20} \mathrm{H}_{24} \mathrm{ClN}_{2}(\mathrm{M}+\mathrm{H})^{+}$calcd.; 327.1628 observed; 327.1602.

$N, N^{\prime}$-bis(1-adamantylmethyl)quinoline-2,8-diamine (4). Obtained as the second product in the synthesis of compound 2b. Eluent petroleum ether- $\mathrm{CH}_{2} \mathrm{Cl}_{2}$ 1:1. Yield $16 \mathrm{mg}$ (26\%), yellowish viscous oil. ${ }^{1} \mathrm{H}-\mathrm{NMR}\left(\mathrm{CDCl}_{3}\right) \delta$ 1.59-1.79 (m, 24H), 1.98 (br.s, 3H), 2.03 (br.s, 3H), 2.95 (d, J=5.3 Hz, 2H), 3.28 (d, $J=6.4 \mathrm{~Hz}, 2 \mathrm{H}), 4.66$ (br.s, 1H), 5.97 (br.s, 1H), 6.54-6.61 (m, 2H), 6.83 (d, J=8.0 Hz, 1H), 7.05 $\left(\mathrm{dd}, J_{o b s}=7.5,7.5 \mathrm{~Hz}, 1 \mathrm{H}\right), 7.69(\mathrm{~d}, J=8.6 \mathrm{~Hz}, 1 \mathrm{H}) .{ }^{13} \mathrm{C}-\mathrm{NMR}\left(\mathrm{CDCl}_{3}\right) \delta 28.4(3 \mathrm{C}), 28.5(3 \mathrm{C}), 34.0$ (1C), 34.7 (1C), 37.1 (3C), 37.2 (3C), 40.8 (3C), 40.9 (3C), 53.1 (1C), 55.9 (1C), 105.0 (1C), 111.4 
(1C), 113.4 (1C), 121.3 (1C), 122.6 (1C), 137.5 (1C), 143.2 (1C), 144.3 (1C), 155.5 (1C). HRMS (MALDI-TOF): $\mathrm{C}_{31} \mathrm{H}_{42} \mathrm{~N}_{3}(\mathrm{M}+\mathrm{H})^{+}$calcd.; 456.3379 observed; 456.3425 .

$\mathrm{N}$-[2-(1-Adamantyl)-1-methylethyl]-8-chloroquinolin-2-amine (2c). Obtained from 2,8-dichloroquinoline (50 mg), amine 1c (49 mg) in the presence of Pd(dba) 2 (6 mg), BINAP (7 mg) and $t$-BuONa (36 mg). Eluent petroluem ether- $\mathrm{CH}_{2} \mathrm{Cl}_{2}$ 2:1-1:1. Yield $52 \mathrm{mg}$ (56\%), yellowish viscous oil. ${ }^{1} \mathrm{H}-\mathrm{NMR}\left(\mathrm{CDCl}_{3}\right)$ $\delta 1.27(\mathrm{~d}, J=6.4 \mathrm{~Hz}, 3 \mathrm{H}), 1.30(\mathrm{dd}, J=14.4,4.2 \mathrm{~Hz}, 1 \mathrm{H}), 1.39(\mathrm{dd}, J=14.4,7.3 \mathrm{~Hz}, 1 \mathrm{H}), 1.54-1.69$ (m, 12H), 1.92 (br.s, 3H), 4.33 (br.s, 1H), 4.71 (br.s, 1H), 6.62 (d, $J=8.8 \mathrm{~Hz}, 1 \mathrm{H}), 7.06$ (dd, $J_{o b s}=7.7$, $7.7 \mathrm{~Hz}, 1 \mathrm{H}), 7.45$ (dd, $J=7.8,1.0 \mathrm{~Hz}, 1 \mathrm{H}), 7.62(\mathrm{dd}, J=7.5,1.0 \mathrm{~Hz}, 1 \mathrm{H}), 7.77(\mathrm{~d}, J=8.8 \mathrm{~Hz}, 1 \mathrm{H})$. ${ }^{13} \mathrm{C}-\mathrm{NMR}\left(\mathrm{CDCl}_{3}\right) \delta 23.7(1 \mathrm{C}), 28.6(3 \mathrm{C}), 32.5$ (1C), $37.0(3 \mathrm{C}), 42.9(4 \mathrm{C}), 52.8(1 \mathrm{C}), 111.7(1 \mathrm{C})$, 121.2 (1C), 124.3 (1C), 126.3 (1C), 129.5 (1C), 129.9 (1C), 137.4 (1C), 156.2 (1C), one quaternary carbon atom was not assigned due to line broadening. HRMS (MALDI-TOF): $\mathrm{C}_{22} \mathrm{H}_{28} \mathrm{ClN} \mathrm{N}_{2}(\mathrm{M}+\mathrm{H})^{+}$ calcd.; 355.1941 observed; 355.1917.

$N$-[1-Adamantyl(phenyl)methyl]-8-chloroquinolin-2-amine (2d). Obtained from 2,8-dichloroquinoline $(50 \mathrm{mg})$, amine 1d $(60 \mathrm{mg})$ in the presence of Pd(dba) $2(6 \mathrm{mg})$, DavePhos $(5 \mathrm{mg})$ and $t$-BuONa (30 mg). Eluent petroleum ether- $\mathrm{CH}_{2} \mathrm{Cl}_{2} 2: 1$. Yield $42 \mathrm{mg}(42 \%)$, yellowish viscous oil. ${ }^{1} \mathrm{H}-\mathrm{NMR}\left(\mathrm{CDCl}_{3}\right) \delta$ 1.52-1.62 (m, 6H), 1.63-1.72 (m, 3H), 1.72-1.80 (m, 3H), 2.00 (br.s, 3H), 4.52 (br.s, 1H), 5.77 (br.s, $1 \mathrm{H}), 6.57(\mathrm{~d}, J=8.8 \mathrm{~Hz}, 1 \mathrm{H}), 7.03\left(\mathrm{dd}, J_{o b s}=7.7,7.7 \mathrm{~Hz}, 1 \mathrm{H}\right), 7.16-7.33(\mathrm{~m}, 5 \mathrm{H}), 7.40(\mathrm{~d}, J=7.3 \mathrm{~Hz}$, $1 \mathrm{H}), 7.59(\mathrm{~d}, J=7.3 \mathrm{~Hz}, 1 \mathrm{H}), 7.68(\mathrm{~d}, J=8.8 \mathrm{~Hz}, 1 \mathrm{H}) .{ }^{13} \mathrm{C}-\mathrm{NMR}\left(\mathrm{CDCl}_{3}\right) \delta 28.4(3 \mathrm{C}), 36.5(1 \mathrm{C}), 36.8$ (3C), 39.2 (3C), 66.0 (1C), 110.9 (1C), 121.4 (1C), 124.5 (1C), 126.3 (1C), 126.9 (1C), 127.6 (2C), 128.8 (2C), 129.2 (1C), 129.5 (1C), 137.6 (1C), 139.8 (1C), 144.3 (1C), 154.4 (1C). HRMS (MALDITOF): $\mathrm{C}_{26} \mathrm{H}_{28} \mathrm{ClN}_{2}(\mathrm{M}+\mathrm{H})^{+}$calcd.; 403.1941 observed; 403.1960.

$\mathrm{N}$-[2-(1-Adamantyloxy)ethyl]-8-chloroquinolin-4-amine (5a). Obtained from 4,8-dichloroquinoline (50 mg), amine 1a (49 mg) in the presence of $\mathrm{Pd}(\mathrm{dba})_{2}(6 \mathrm{mg})$, BINAP $(7 \mathrm{mg})$ and $t$-BuONa $(36 \mathrm{mg})$. Eluent $\mathrm{CH}_{2} \mathrm{Cl}_{2} / \mathrm{MeOH}$ 100:1-50:1. Yield $69 \mathrm{mg}$ (77\%), beige crystalline powder, m.p. $225-227{ }^{\circ} \mathrm{C} .{ }^{1} \mathrm{H}-\mathrm{NMR}$ $\left(\mathrm{CDCl}_{3}\right) \delta 1.55-1.68(\mathrm{~m}, 6 \mathrm{H}), 1.74-1.77(\mathrm{~m}, 6 \mathrm{H}), 2.15$ (br.s, 3H), $3.40(\mathrm{q}, J=5.1 \mathrm{~Hz}, 2 \mathrm{H}), 3.73$ (t, $J=5.2 \mathrm{~Hz}, 2 \mathrm{H}), 5.56$ (br.s, $1 \mathrm{H}), 6.45(\mathrm{~d}, J=5.4 \mathrm{~Hz}, 1 \mathrm{H}), 7.31\left(\mathrm{dd}, J_{o b s}=8.0,8.0 \mathrm{~Hz}, 1 \mathrm{H}\right), 7.64(\mathrm{~d}$, $J=8.5 \mathrm{~Hz}, 1 \mathrm{H}), 7.74(\mathrm{~d}, J=7.5 \mathrm{~Hz}, 1 \mathrm{H}), 8.64(\mathrm{~d}, J=5.4 \mathrm{~Hz}, 1 \mathrm{H}) .{ }^{13} \mathrm{C}-\mathrm{NMR}\left(\mathrm{CDCl}_{3}\right) \delta 30.4(3 \mathrm{C})$, 36.3 (3C), 41.6 (3C), 43.4 (1C), 57.5 (1C), 72.8 (1C), 99.7 (1C), 118.5 (1C), 120.2 (1C), 124.1 (1C), 129.2 (1C), 133.8 (1C), 144.8 (1C), 150.1 (1C), 151.4 (1C). HRMS (MALDI-TOF): $\mathrm{C}_{21} \mathrm{H}_{26} \mathrm{ClN}_{2} \mathrm{O}$ $(\mathrm{M}+\mathrm{H})^{+}$calcd.; 357.1734 observed; 357.1698 .

$N, N^{\prime}$-bis [2-(1-Adamantyloxy)ethyl]quinoline-4,8-diamine (6a). Obtained from 4,8-dichloroquinoline $(50 \mathrm{mg})$, amine 1a (195 mg) in the presence of Pd(dba) $(12 \mathrm{mg})$, DavePhos $(9 \mathrm{mg})$ and $t$-BuONa (60 mg). Eluent $\mathrm{CH}_{2} \mathrm{Cl}_{2} / \mathrm{MeOH} 50: 1$. Yield $68 \mathrm{mg}(52 \%)$, yellowish viscous oil. ${ }^{1} \mathrm{H}-\mathrm{NMR}\left(\mathrm{CDCl}_{3}\right) \delta 1.55-1.75$ (m, 12H), 1.77 (br.s, 12H), 2.13 (br.s, 3H), 2.16 (br.s, 3H), 3.37-3.46 (m, 4H), 3.73 (t, J=5.8 Hz, 4H), 5.44 (br.s, 1H), 5.58 (br.s, 1H), 6.41 (d, $J=5.3 \mathrm{~Hz}, 1 \mathrm{H}), 6.67$ (d, $J=7.7 \mathrm{~Hz}, 1 \mathrm{H}), 6.90$ (d, $J=8.5 \mathrm{~Hz}$, $1 \mathrm{H}), 7.27$ (dd, $\left.J_{o b s}=8.1,8.1 \mathrm{~Hz}, 1 \mathrm{H}\right), 8.37$ (d, $\left.J=5.3 \mathrm{~Hz}, 1 \mathrm{H}\right)$. HRMS (MALDI-TOF): $\mathrm{C}_{33} \mathrm{H}_{46} \mathrm{~N}_{3} \mathrm{O}_{2}$ $(\mathrm{M}+\mathrm{H})^{+}$calcd.; 516.3590 observed; 516.3561 . 
$\mathrm{N}$-(1-Adamantylmethyl)-8-chloroquinolin-4-amine (5b). Obtained from 4,8-dichloroquinoline (50 mg), amine $1 \mathbf{b}(41 \mathrm{mg})$ in the presence of $\mathrm{Pd}(\mathrm{dba})_{2}(6 \mathrm{mg})$, BINAP $(7 \mathrm{mg})$ and $t$-BuONa $(36 \mathrm{mg})$. Eluent $\mathrm{CH}_{2} \mathrm{Cl}_{2} / \mathrm{MeOH}$ 100:1. Yield $56 \mathrm{mg}$ (67\%), beige crystalline powder, m.p. 210-212 ${ }^{\circ} \mathrm{C}$. ${ }^{1} \mathrm{H}-\mathrm{NMR}$ $\left(\mathrm{CDCl}_{3}\right) \delta 1.58-1.61(\mathrm{~m}, 6 \mathrm{H}), 1.61-1.76(\mathrm{~m}, 6 \mathrm{H}), 2.00$ (br.s, 3H), $2.69(\mathrm{~d}, J=5.7 \mathrm{~Hz}, 2 \mathrm{H}), 5.11(\mathrm{t}$, $J=5.7 \mathrm{~Hz}, 1 \mathrm{H}), 6.47(\mathrm{~d}, J=5.4 \mathrm{~Hz}, 1 \mathrm{H}), 7.27(\mathrm{dd}, J=8.6,7.6 \mathrm{~Hz}, 1 \mathrm{H}), 7.63(\mathrm{dd}, J=8.6,1.1 \mathrm{~Hz}, 1 \mathrm{H})$, $7.71(\mathrm{dd}, J=7.6,1.1 \mathrm{~Hz}, 1 \mathrm{H}), 8.60(\mathrm{~d}, J=5.4 \mathrm{~Hz}, 1 \mathrm{H}) .{ }^{13} \mathrm{C}-\mathrm{NMR}\left(\mathrm{CDCl}_{3}\right) \delta 28.1(3 \mathrm{C}), 34.0(1 \mathrm{C}), 36.8$ (3C), 40.6 (3C), 55.0 (1C), 99.4 (1C), 118.2 (1C), 119.9 (1C), 123.9 (1C), 129.1 (1C), 133.9 (1C), 144.8 (1C), 150.5 (1C), 151.4 (1C). HRMS (MALDI-TOF): $\mathrm{C}_{20} \mathrm{H}_{24} \mathrm{ClN}_{2}(\mathrm{M}+\mathrm{H})^{+}$calcd.; 327.1628 observed; 327.1654.

N-[2-(1-adamantyl)-1-methylethyl]-8-chloroquinolin-4-amine (5c). Obtained from 4,8-dichloroquinoline (50 mg), amine 1c (49 mg) in the presence of Pd(dba) $)_{2}(6 \mathrm{mg})$, BINAP (7 mg) and $t$-BuONa (36 mg). Eluent $\mathrm{CH}_{2} \mathrm{Cl}_{2} / \mathrm{MeOH}$ 100:1-50:1. Yield $75 \mathrm{mg}(84 \%)$, yellowish viscous oil. ${ }^{1} \mathrm{H}-\mathrm{NMR}\left(\mathrm{CDCl}_{3}\right) \delta$ 1.25 (d, $J=6.4 \mathrm{~Hz}, 3 \mathrm{H}), 1.38$ (dd, $J=14.6,3.9 \mathrm{~Hz}, 1 \mathrm{H}), 1.45$ (dd, $J=14.6,7.6 \mathrm{~Hz}, 1 \mathrm{H}), 1.53$ (br.s, $6 \mathrm{H}), 1.54-1.67$ (m, 6H), 1.90 (br.s, 3H), 3.78-3.87 (m, 1H), 4.85 (d, J= 7.3 Hz, 1H), 6.48 (d, J=5.5 Hz, $1 \mathrm{H}), 7.28(\mathrm{dd}, J=8.6,7.5 \mathrm{~Hz}, 1 \mathrm{H}), 7.60(\mathrm{dd}, J=8.6,1.1 \mathrm{~Hz}, 1 \mathrm{H}), 7.72(\mathrm{dd}, J=7.5,1.1 \mathrm{~Hz}, 1 \mathrm{H}), 8.64$ $(\mathrm{d}, J=5.5 \mathrm{~Hz}, 1 \mathrm{H}) .{ }^{13} \mathrm{C}-\mathrm{NMR}\left(\mathrm{CDCl}_{3}\right) \delta 22.4(1 \mathrm{C}), 28.5(3 \mathrm{C}), 32.5(1 \mathrm{C}), 36.8(3 \mathrm{C}), 42.9(3 \mathrm{C}), 44.2$ (1C), 52.6 (1C), 99.2 (1C), 118.2 (1C), 120.0 (1C), 124.0 (1C), 129.2 (1C), 133.8 (1C), 145.0 (1C), 148.5 (1C), 151.4 (1C). HRMS (MALDI-TOF): $\mathrm{C}_{22} \mathrm{H}_{28} \mathrm{ClN}_{2}(\mathrm{M}+\mathrm{H})^{+}$calcd.; 355.1941 observed; 355.1978.

$N$-[1-Adamantyl(phenyl)methyl]-8-chloroquinolin-4-amine (5d). Obtained from 4,8-dichloroquinoline $(50 \mathrm{mg})$, amine 1d (60 mg) in the presence of Pd(dba) 2 (6 mg), BINAP (7 mg) and $t$-BuONa (36 mg). Eluent $\mathrm{CH}_{2} \mathrm{Cl}_{2} / \mathrm{MeOH}$ 100:1. Yield $70 \mathrm{mg}(70 \%)$, beige crystalline powder, m.p. $171-173{ }^{\circ} \mathrm{C} .{ }^{1} \mathrm{H}-\mathrm{NMR}$ $\left(\mathrm{CDCl}_{3}\right) \delta 1.55-1.67(\mathrm{~m}, 6 \mathrm{H}), 1.68-1.81(\mathrm{~m}, 6 \mathrm{H}), 2.04$ (br.s, 3H), $4.08(\mathrm{~d}, J=6.2 \mathrm{~Hz}, 1 \mathrm{H}), 5.77(\mathrm{~d}$, $J=6.2 \mathrm{~Hz}, 1 \mathrm{H}), 6.19(\mathrm{~d}, J=5.4 \mathrm{~Hz}, 1 \mathrm{H}), 7.20-7.32(\mathrm{~m}, 5 \mathrm{H}), 7.38\left(\mathrm{dd}, J_{o b s}=7.9,7.9 \mathrm{~Hz}, 1 \mathrm{H}\right), 7.76(\mathrm{~d}$, $J=7.6 \mathrm{~Hz}, 1 \mathrm{H}), 7.79(\mathrm{~d}, J=8.3 \mathrm{~Hz}, 1 \mathrm{H}), 8.44(\mathrm{~d}, J=5.4 \mathrm{~Hz}, 1 \mathrm{H}) .{ }^{13} \mathrm{C}-\mathrm{NMR}\left(\mathrm{CDCl}_{3}\right) \delta 28.2(3 \mathrm{C})$, 36.5 (1C), 36.7 (3C), 39.3 (3C), 67.2 (1C), 100.9 (1C), 117.7 (1C), 120.2 (1C), 124.2 (1C), 127.4 (1C), 127.9 (2C), 128.3 (2C), 129.1 (1C), 134.1 (1C), 137.9 (1C), 144.7 (1C), 149.0 (1C), 151.3 (1C). HRMS (MALDI-TOF): $\mathrm{C}_{26} \mathrm{H}_{28} \mathrm{ClN}_{2}(\mathrm{M}+\mathrm{H})^{+}$calcd.; 403.1941 observed; 403.1930.

$N, N$ '-bis[1-Adamantyl(phenyl)methyl]quinoline-4,8-diamine (6d). Obtained from 4,8-dichloroquinoline $(50 \mathrm{mg})$, amine 1d (180 mg) in the presence of $\mathrm{Pd}(\mathrm{dba})_{2}(12 \mathrm{mg})$, DavePhos $(9 \mathrm{mg})$ and $t$-BuONa $(72 \mathrm{mg})$. Eluent petroleum ether- $\mathrm{CH}_{2} \mathrm{Cl}_{2}$ 1:1. Yield $75 \mathrm{mg}(45 \%)$, beige crystalline powder, m.p. 175-177 ${ }^{\circ} \mathrm{C} .{ }^{1} \mathrm{H}-\mathrm{NMR}\left(\mathrm{CDCl}_{3}\right) \delta 1.50-1.83(\mathrm{~m}, 24 \mathrm{H}), 1.95-2.04(\mathrm{~m}, 6 \mathrm{H}), 4.00(\mathrm{~d}, J=6.6 \mathrm{~Hz})+4.02$ $(\mathrm{d}, J=6.7 \mathrm{~Hz})(1 \mathrm{H}$ for two diastereomers $), 4.06(\mathrm{~d}, J=5.0 \mathrm{~Hz}, 1 \mathrm{H}), 5.59(\mathrm{~d}, J=5.2 \mathrm{~Hz}, 1 \mathrm{H}), 6.11(\mathrm{~d}$, $J=5.2 \mathrm{~Hz}, 1 \mathrm{H}), 6.27(\mathrm{~d}, J=7.5 \mathrm{~Hz}, 1 \mathrm{H}), 6.91(\mathrm{~d}, J=8.3 \mathrm{~Hz}, 1 \mathrm{H}), 7.06-7.35(\mathrm{~m}, 12 \mathrm{H}), 8.22(\mathrm{~d}$, $J=5.2 \mathrm{~Hz}, 1 \mathrm{H}) .{ }^{13} \mathrm{C}-\mathrm{NMR}\left(\mathrm{CDCl}_{3}\right) \delta 28.3(3 \mathrm{C}), 28.5(3 \mathrm{C}), 36.6(1 \mathrm{C}), 36.7(1 \mathrm{C}), 36.8(3 \mathrm{C}), 37.0(3 \mathrm{C})$, 39.3 (6C), 67.1 (1C), 67.8 (1C), $100.4+100.5$ (1C for two diastereomers), 103.9 (1C), 105.0 (1C), 125.7 (1C), 126.6 (1C), 127.1 (1C), 127.4 (2C), 127.8 (2C), 128.4 (2C), 128.8 (2C), 138.1 (1C), 138.7 (1C), 140.3 (1C), 144.9 (1C), 145.0 (1C), 147.4 (1C), 148.7 (1C). HRMS (MALDI-TOF): $\mathrm{C}_{43} \mathrm{H}_{50} \mathrm{~N}_{3}$ $(\mathrm{M}+\mathrm{H})^{+}$calcd.; 608.4005 observed; 608.3969 . 
$\mathrm{N}$-[2-(1-adamantyloxy)ethyl]-7-chloroquinolin-4-amine (7a). Obtained from 4,7-dichloroquinoline (50 mg), amine 1a (49 mg) in the presence of $\mathrm{Pd}(\mathrm{dba})_{2}(6 \mathrm{mg})$, BINAP $(7 \mathrm{mg})$ and $t$-BuONa $(36 \mathrm{mg})$. Eluent $\mathrm{CH}_{2} \mathrm{Cl}_{2} / \mathrm{MeOH}$ 50:1-35:1. Yield $46 \mathrm{mg}$ (52\%), beige crystalline powder, m.p. 173-175 ${ }^{\circ} \mathrm{C}$. ${ }^{1} \mathrm{H}-\mathrm{NMR}$ $\left(\mathrm{CDCl}_{3}\right) \delta 1.55-1.67(\mathrm{~m}, 6 \mathrm{H}), 1.74-1.77(\mathrm{~m}, 6 \mathrm{H}), 2.15$ (br.s, 3H), $3.39(\mathrm{q}, J=5.1 \mathrm{~Hz}, 2 \mathrm{H}), 3.72(\mathrm{t}$, $J=5.2 \mathrm{~Hz}, 2 \mathrm{H}), 5.54$ (br.s, $1 \mathrm{H}), 6.38(\mathrm{~d}, J=5.3 \mathrm{~Hz}, 1 \mathrm{H}), 7.35(\mathrm{dd}, J=9.0,2.2 \mathrm{~Hz}, 1 \mathrm{H}), 7.64(\mathrm{~d}$, $J=9.0 \mathrm{~Hz}, 1 \mathrm{H}), 7.93(\mathrm{~d}, J=2.2 \mathrm{~Hz}, 1 \mathrm{H}), 8.50(\mathrm{~d}, J=5.3 \mathrm{~Hz}, 1 \mathrm{H}) .{ }^{13} \mathrm{C}-\mathrm{NMR}\left(\mathrm{CDCl}_{3}\right) \delta 30.4(3 \mathrm{C})$, 36.3 (3C), 41.6 (3C), 43.3 (1C), 57.6 (1C), 72.8 (1C), 99.2 (1C), 117.3 (1C), 121.0 (1C), 125.3 (1C), 128.6 (1C), 134.8 (1C), 149.0 (1C), 149.9 (1C), 151.9 (1C). HRMS (MALDI-TOF): $\mathrm{C}_{21} \mathrm{H}_{26} \mathrm{ClN}_{2} \mathrm{O}$ $(\mathrm{M}+\mathrm{H})^{+}$calcd.; 357.1734 observed; 357.1715 .

$N, N^{\prime}$-bis [2-(1-Adamantyloxy)ethyl]quinoline-4,7-diamine (8a). Obtained from 4,7-dichloroquinoline $(50 \mathrm{mg})$, amine 1a (195 mg) in the presence of Pd(dba) $2(12 \mathrm{mg})$, DavePhos $(9 \mathrm{mg})$ and $t$-BuONa (60 mg). Eluent $\mathrm{CH}_{2} \mathrm{Cl}_{2} / \mathrm{MeOH}$ 20:1-3:1. Yield $92 \mathrm{mg}$ (71\%), beige crystalline powder, m.p. 160-162 ${ }^{\circ} \mathrm{C}$. ${ }^{1} \mathrm{H}-\mathrm{NMR}\left(\mathrm{CDCl}_{3}\right) \delta 1.53-1.66(\mathrm{~m}, 12 \mathrm{H}), 1.72$ (br.s, 12H), 2.12 (br.s, 6H), 3.31 (q, $\left.J=4.5 \mathrm{~Hz}, 2 \mathrm{H}\right), 3.42$ $(\mathrm{q}, J=5.1 \mathrm{~Hz}, 2 \mathrm{H}), 3.63(\mathrm{t}, J=5.0 \mathrm{~Hz}, 2 \mathrm{H}), 3.70$ (t, $J=5.4 \mathrm{~Hz}, 2 \mathrm{H}), 4.59$ (br.s, $1 \mathrm{H}), 6.16$ (br.s, $1 \mathrm{H})$, $6.22(\mathrm{~d}, J=5.9 \mathrm{~Hz}, 1 \mathrm{H}), 6.79(\mathrm{dd}, J=9.0,1.9 \mathrm{~Hz}, 1 \mathrm{H}), 6.94(\mathrm{~d}, J=1.9 \mathrm{~Hz}, 1 \mathrm{H}), 7.62(\mathrm{~d}, J=9.0 \mathrm{~Hz}$, $1 \mathrm{H}), 8.21(\mathrm{~d}, J=5.9 \mathrm{~Hz}, 1 \mathrm{H}) .{ }^{13} \mathrm{C}-\mathrm{NMR}\left(\mathrm{CDCl}_{3}\right) \delta 30.4(6 \mathrm{C}), 36.3(6 \mathrm{C}), 41.5(6 \mathrm{C}), 43.4(1 \mathrm{C}), 43.9$ (1C), 57.9 (2C), 72.4 (1C), 72.7 (1C), 99.7 (1C), 104.0 (1C), 110.6 (1C), 116.4 (1C), 121.0 (1C), 148.0 (1C), 148.1 (1C), 149.6 (1C), 151.2 (1C). HRMS (MALDI-TOF): $\mathrm{C}_{33} \mathrm{H}_{46} \mathrm{~N}_{3} \mathrm{O}_{2}(\mathrm{M}+\mathrm{H})^{+}$calcd.; 516.3590 observed; 516.3634 .

$\mathrm{N}$-(1-Adamantylmethyl)-7-chloroquinolin-4-amine (7b). Obtained from 4,7-dichloroquinoline (50 mg), amine $\mathbf{1 b}(41 \mathrm{mg})$ in the presence of $\mathrm{Pd}(\mathrm{dba})_{2}(6 \mathrm{mg})$, BINAP $(7 \mathrm{mg})$ and $t$-BuONa $(36 \mathrm{mg})$. Eluent $\mathrm{CH}_{2} \mathrm{Cl}_{2} / \mathrm{MeOH}$ 100:1-50:1. Yield $50 \mathrm{mg}$ (61\%), beige crystalline powder, m.p. $225-227{ }^{\circ} \mathrm{C} .{ }^{1} \mathrm{H}-\mathrm{NMR}$ $\left(\mathrm{CD}_{3} \mathrm{OD}\right) \delta 1.65$ (br.s, 6H), 1.65-1.78 (m, 6H), 1.97 (br.s, 3H), 3.09 (s, 2H), 6.60 (d, J=5.8 Hz, 1H), $7.39(\mathrm{dd}, J=9.0,2.1 \mathrm{~Hz}, 1 \mathrm{H}), 7.76(\mathrm{~d}, J=2.1 \mathrm{~Hz}, 1 \mathrm{H}), 8.13(\mathrm{~d}, J=9.0 \mathrm{~Hz}, 1 \mathrm{H}), 8.30(\mathrm{~d}$, $J=5.8 \mathrm{~Hz}, 1 \mathrm{H}), \mathrm{NH}$ proton was not observed. ${ }^{13} \mathrm{C}-\mathrm{NMR}\left(\mathrm{CD}_{3} \mathrm{OD}\right) \delta 29.8(3 \mathrm{C}), 36.7(1 \mathrm{C}), 38.0(3 \mathrm{C})$, 41.7 (3C), 55.5 (1C), 100.0 (1C), 118.6 (1C), 124.2 (1C), 125.9 (1C), 127.5 (1C), 136.3 (1C), 149.7 (1C), 152.2 (1C), 153.8 (1C). HRMS (MALDI-TOF): $\mathrm{C}_{20} \mathrm{H}_{24} \mathrm{ClN}_{2}(\mathrm{M}+\mathrm{H})^{+}$calcd.; 327.1628 observed; 327.1649.

N-[2-(1-Adamantyl)-1-methylethyl]-7-chloroquinolin-4-amine (7c). Obtained from 4,7-dichloroquinoline (50 mg), amine 1c (49 mg) in the presence of Pd(dba) 2 (6 mg), BINAP (7 mg) and $t$-BuONa (36 mg). Eluent $\mathrm{CH}_{2} \mathrm{Cl}_{2} / \mathrm{MeOH}$ 50:1. Yield $73 \mathrm{mg}$ (79\%), beige crystalline powder, m.p. 207-209 ${ }^{\circ} \mathrm{C}$. ${ }^{1} \mathrm{H}-\mathrm{NMR}$ $\left(\mathrm{CDCl}_{3}\right) \delta 1.26(\mathrm{~d}, J=6.2 \mathrm{~Hz}, 3 \mathrm{H}), 1.39(\mathrm{dd}, J=14.8,3.9 \mathrm{~Hz}, 1 \mathrm{H}), 1.46(\mathrm{dd}, J=14.8,7.6 \mathrm{~Hz}, 1 \mathrm{H}), 1.54$ (br.s, 6H), 1.54-1.69 (m, 6H), 1.91 (br.s, 3H), 3.78-3.87 (m, 1H), 4.78 (d, J= $7.2 \mathrm{~Hz}, 1 \mathrm{H}), 6.42$ (d, $J=5.5 \mathrm{~Hz}, 1 \mathrm{H}), 7.33(\mathrm{dd}, J=9.1,2.2 \mathrm{~Hz}, 1 \mathrm{H}), 7.60(\mathrm{~d}, J=9.1 \mathrm{~Hz}, 1 \mathrm{H}), 7.93(\mathrm{~d}, J=2.2 \mathrm{~Hz}, 1 \mathrm{H}), 8.51$ $(\mathrm{d}, J=5.5 \mathrm{~Hz}, 1 \mathrm{H}) .{ }^{13} \mathrm{C}-\mathrm{NMR}\left(\mathrm{CDCl}_{3}\right) \delta 22.4(1 \mathrm{C}), 28.5(3 \mathrm{C}), 32.5(1 \mathrm{C}), 36.8(3 \mathrm{C}), 43.0(3 \mathrm{C}), 44.1$ (1C), 52.6 (1C), 98.8 (1C), 117.2 (1C), 120.7 (1C), 125.1 (1C), 128.8 (1C), 134.8 (1C), 148.2 (1C), 149.3 (1C), 152.0 (1C). HRMS (MALDI-TOF): $\mathrm{C}_{22} \mathrm{H}_{28} \mathrm{ClN}_{2}(\mathrm{M}+\mathrm{H})^{+}$calcd.; 355.1941 observed; 355.1904.

$N$-[1-Adamantyl(phenyl)methyl]-7-chloroquinolin-4-amine (7d). Obtained from 4,7-dichloroquinoline $(50 \mathrm{mg})$, amine 1d (60 mg) in the presence of Pd(dba) 2 (6 mg), BINAP (7 mg) and $t$-BuONa (36 mg). Eluent $\mathrm{CH}_{2} \mathrm{Cl}_{2} / \mathrm{MeOH}$ 100:1. Yield $77 \mathrm{mg}$ (77\%), yellowish viscous oil. ${ }^{1} \mathrm{H}-\mathrm{NMR}\left(\mathrm{CDCl}_{3}\right) \delta 1.54-1.66$ 
(m, 6H), 1.68-1.80 (m, 6H), 2.03 (br.s, 3H), 4.07 (d, $J=6.2 \mathrm{~Hz}, 1 \mathrm{H}), 5.70$ (d, $J=6.2 \mathrm{~Hz}, 1 \mathrm{H}), 6.13$ (d, $J=5.3 \mathrm{~Hz}, 1 \mathrm{H}), 7.23-7.33(\mathrm{~m}, 5 \mathrm{H}), 7.41(\mathrm{dd}, J=8.8,1.9 \mathrm{~Hz}, 1 \mathrm{H}), 7.79(\mathrm{~d}, J=8.8 \mathrm{~Hz}, 1 \mathrm{H}), 7.93(\mathrm{~d}$, $J=1.9 \mathrm{~Hz}, 1 \mathrm{H}), 8.31(\mathrm{~d}, J=5.3 \mathrm{~Hz}, 1 \mathrm{H}) .{ }^{13} \mathrm{C}-\mathrm{NMR}\left(\mathrm{CDCl}_{3}\right) \delta 28.2(3 \mathrm{C}), 36.5(1 \mathrm{C}), 36.7$ (3C), 39.3 (3C), 67.1 (1C), 100.4 (1C), 117.4 (1C), 120.4 (1C), 125.3 (1C), 127.4 (1C), 127.9 (2C), 128.7 (2C), 129.0 (1C), 134.6 (1C), 138.0 (1C), 148.7 (1C), 148.9 (1C), 151.9 (1C). HRMS (MALDI-TOF): $\mathrm{C}_{26} \mathrm{H}_{28} \mathrm{ClN}_{2}(\mathrm{M}+\mathrm{H})^{+}$calcd.; 403.1941 observed; 403.1958.

$N, N^{\prime}$-bis[1-Adamantyl(phenyl)methyl]quinoline-4,7-diamine (8d). Obtained from 4,7-dichloroquinoline $(50 \mathrm{mg})$, amine 1d $(180 \mathrm{mg})$ in the presence of $\mathrm{Pd}(\mathrm{dba})_{2}(12 \mathrm{mg})$, BINAP (14 mg) and $t$-BuONa (72 mg). Eluent $\mathrm{CH}_{2} \mathrm{Cl}_{2} / \mathrm{MeOH} 35: 20: 1$. Yield $88 \mathrm{mg}$ (58\%), beige crystalline powder, m.p. $240-242{ }^{\circ} \mathrm{C}$. ${ }^{1} \mathrm{H}-\mathrm{NMR}\left(\mathrm{CDCl}_{3}\right) \delta 1.48-1.63(\mathrm{~m}, 12 \mathrm{H}), 1.63-1.76$ (m, 12H), 1.99 (br.s, 6H), 4.00-4.06 (2H), 4.74 (d, $J=6.7 \mathrm{~Hz}, 1 \mathrm{H}), 5.54(\mathrm{~d}, J=6.1 \mathrm{~Hz}), 5.85(\mathrm{~d}, J=5.4 \mathrm{~Hz})+5.86(\mathrm{~d}, J=5.4 \mathrm{~Hz})(1 \mathrm{H}$ for two diastereomers), 6.81 (br.s, $1 \mathrm{H}), 6.85(\mathrm{~d}, J=9.0 \mathrm{~Hz}, 1 \mathrm{H}), 7.14-7.28(\mathrm{~m}, 10 \mathrm{H}), 7.56(\mathrm{~d}, J=9.0 \mathrm{~Hz}, 1 \mathrm{H})$, $8.07(\mathrm{~d}, J=5.4 \mathrm{~Hz}, 1 \mathrm{H}) .{ }^{13} \mathrm{C}-\mathrm{NMR}\left(\mathrm{CDCl}_{3}\right) \delta 28.3(3 \mathrm{C}), 28.4(3 \mathrm{C}), 36.5(2 \mathrm{C}), 36.8(3 \mathrm{C}), 36.8(3 \mathrm{C})$, 39.2 (3C), 39.3 (3C), 66.9 (1C), $67.6(1 \mathrm{C}), 97.1+97.8$ (1C for two diastereomers), 107.1 (1C), 111.0 (1C), 115.7 (1C), 119.6 (1C), 126.9 (1C), 127.2 (1C), 127.7 (2C), 127.8 (2C), 128.3 (2C), 128.6 (2C), 138.5 (1C), 138.6 (1C), 139.5 (1C), 148.4 (1C), 149.1 (1C), 150.3 (1C). HRMS (MALDI-TOF): $\mathrm{C}_{43} \mathrm{H}_{50} \mathrm{~N}_{3}(\mathrm{M}+\mathrm{H})^{+}$calcd.; 608.4005 observed; 608.3980 .

\section{Conclusions}

To sum up, we have investigated the Pd-catalyzed amination reactions of isomeric chloroquinolines with several adamantane-containing amines and found out that 4,8- and 4,7-dichloroquinolines provided the best yields of the mono- and diamination reaction products due to a difference in the reactivity of the chlorine atoms. 2,8-Dichloroquinoline proved to be a more problematic substrate, especially in the case of the diamination process, and the application of 2,6-dichloroquinoline led only to complicated mixtures of unidentified products. The monoamination reactions were successfully performed using 1 equiv. of the amine and BINAP as a ligand, while diamination processes demanded the use of 3-4 equiv. of the amines and DavePhos ligand in the majority of cases. There was no pronounced dependence of the yields of the monoamination products on the bulkiness of the amine used, but the diamination processes were more successful for a less sterically hindered amine 1a compared to the most hindered amine $\mathbf{1 d}$.

\section{Acknowledgments}

This work was supported by the RFBR grant N 10-03-01108 and by the Russian Academy of Sciences program "Elaboration of the methods for the synthesis of chemical compounds and construction of new materials".

\section{References}

1. Gilligan, B.S.; Veale, J.; Wodak, J. Amantadine hydrochloride in the treatment of Parkinson's Disease. Med. J. Aust. 1970, 2, 634-637. 
2. Dolin, R.; Bentley, D.W. Options for the Control of Influenza. In Proceedings of the Viratek-UCLA Symposium, Keystone, CO, USA, 20-25 April 1985; p. 317.

3. Svenson, T.N. Dopamine release and direct dopamine receptor activation in central nervous system by D-145, an amantadine derivative. Eur. J. Pharmacol. 1973, 34, 232-238.

4. Da Settimo, A.; Marini, A.M.; Primofiore, G.; da Settimo, F. Synthesis and evaluation of aminoadamantane derivatives for in vitro anti-HIV and antitumor activities. Farmacology 1995, 50, 321-326.

5. Ismail, F.M.D.; Dascombe, M.I.; Carrand, P.; North, S.E. An exploration of the structure-activity relationships of 4-aminoquinolines: Novel antimalarials with activity in vivo. J. Pharm. Pharmacol. 1996, 48, 841-850.

6. Koert, G. Adamantylamino quinolines. U.S. Patent 3730956(A), 1 May 1973.

7. Aranyi, P.; Bata, I.; Batory, S.; Boronkay, E.; Bovy, P.; Kapui, Z.; Edit, S.; Szabo, T.; Urban-Szabo, K.; Varga, M. New Compounds. WO 2005021536(A2), 10 March 2003.

8. Surry, D.S.; Buchwald, S.L. Dialkylbiaryl phosphines in Pd-catalyzed amination: A user's guide. Chem. Sci. 2011, 2, 27-50.

9. Surry, D.S.; Buchwald, S.L. Biaryl phosphane ligands in palladium-catalyzed amination. Angew. Chem. Int. Ed. Engl. 2008, 47, 6338-6361.

10. Proutiere, F.; Aufiero, M.; Schoenebeck, F. Reactivity and stability of dinuclear Pd(I) complexes: Studies on the active catalytic species, insights into precatalyst activation and deactivation, and application in highly selective cross-coupling reactions. J. Am. Chem. Soc. 2012, 134, 606-612.

11. Hoi, K.H.; Çalimsiz, S.; Froese, R.D.J.; Hopkinson, A.C.; Organ, M.G. Amination with Pd-NHC complexes: Rate and computational studies on the effects of the oxidative addition partner. Chem. Eur. J. 2011, 17, 3086-3090.

12. Proutiere, F.; Schoenebeck, F. Solvent effect on palladium-catalyzed cross-coupling reactions and implications on the active catalytic species. Angew. Chem. Int. Ed. Engl. 2011, 50, 8192-8195.

13. Jimeno, C.; Christmann, U.; Escudero-Adán, E.C.; Vilar, R.; Pericas, M.A. Studies on the amination of aryl chlorides with a monoligated palladium catalyst: Kinetic evidence for a cooperative mechanism. Chem. Eur. J. 2012, 18, 16510-16516.

14. Deraeve, C.; Maraval, A.; Vendier, L.; Faugeroux, V.; Pitié, M.; Meunier, B. Preparation of new bis(8-aminoquinoline) ligands and comparison with bis(8-hydroxyquinoline) ligands on their ability to chelate $\mathrm{Cu}(\mathrm{II})$ and $\mathrm{Zn}(\mathrm{II})$. Eur. J. Inorg. Chem. 2008, 2008, 5622-5631.

15. Lundgren, R.J.; Sappong-Kumankumah, A.; Stradiotto, M. A highly versatile catalyst system for the cross-coupling of aryl chlorides and amines. Chem. Eur. J. 2010, 16, 1983-1991.

16. Michalik, D.; Kumar, K.; Zapf, A.; Tillack, A.; Arlt, M.; Heinrich, T.; Beller, M. A short and efficient synthesis of $N$-aryl- and $N$-heteroaryl- $N$ '-(arylalkyl)piperazines. Tetrahedron Lett. 2004, 45, 2057-2061.

17. Knight, R.L.; Allen, D.R.; Birch, H.L.; Chapman, G.A.; Galvin, F.C.; Jopling, L.A.; Lock, C.J.; Meissner, J.W.; Owen, D.A.; Raphy, G.; et al. Development of CXCR3 antagonists. Part 4: Discovery of 2-amino-(4-tropinyl)quinolines. Bioorg. Med. Chem. Lett. 2008, 18, 629-633.

18. Rataboul, F.; Zapf, A.; Jackstell, R.; Harkal, S.; Riermeier, T.; Monsees, A.; Dingerdissen, U.; Beller, M. New ligands for a general palladium-catalyzed amination of aryl and heteroaryl chlorides. Chem. Eur. J. 2004, 10, 2983-2990. 
19. Navarro, O.; Marion, N.; Mei, J.; Nolan, P.S. Rapid room temperature Buchwald-Hartwig and Suzuki-Miyaura couplings of heteroaromatic compounds employing low catalyst loadings. Chem. Eur. J. 2006, 12, 5142-5148.

20. Anderson, K.W.; Tundel, R.E.; Ikawa, T.; Altman, R.A.; Buchwald, S.L. Monodentate phosphines provide highly active catalysts for Pd-catalyzed $\mathrm{C}-\mathrm{N}$ bond-forming reactions of heteroaromatic halides/amines and (H)N-heterocycles. Angew. Chem. Int. Ed. Engl. 2006, 45, 6523-6527.

21. Berger, R.; Zhu, C.; Hansen, A.R.; Harper, B.; Chen, Z.; Holt, T.G.; Hubert, J.; Lee, S.J.; Pan, J.; Qian, S.; et al. 2-Substituted piperazine-derived imidazole carboxamides as potent and selective CCK1R agonists for the treatment of obesity. Bioorg. Med. Chem. Lett. 2008, 18, 4833-4837.

22. Nicolas, M.; Ecarnot, E.C.; Navarro, O.; Amoroso, D.; Bell, A.; Nolan, S.P. (IPr)Pd(acac)Cl: An easily synthesized, efficient, and versatile precatalyst for C-N and C-C bond formation. J. Org. Chem. 2006, 71, 3816-3821.

23. Clayden, J.; Hennecke, U. $\alpha$-Pyridylation of chiral amines via urea coupling, lithiation and rearrangement. Org. Lett. 2008, 10, 3567-3570.

24. Tewari, A.; Hein, M.; Zapf, A.; Beller, M. Efficient palladium catalysts for the amination of aryl chlorides: A comparative study on the use of phosphinum salts as precursors to bulky, electron-rich phosphines. Tetrahedron 2005, 61, 9705-9709.

25. Organ, G.M.; Abdel-Hadi, M.; Avola, S.; Dubovyk, I.; Hadei, N.; Assen, B.; Kantchev, E.A.B.; O’Brien, C.J.; Sayah, M.; Valente, C. Pd-catalyzed aryl amination mediated by well defined, $N$-heterocyclic carbene (NHC)-Pd precatalysts, PEPPSI. Chem. Eur. J. 2008, 14, 2443-2452.

26. Serafinowska, H.T.; Blaney, F.E.; Lovell, P.J.; Merlo, G.G.; Scott, C.M.; Smith, P.W.; Starr, K.R.; Watson, J.M. Novel 5-HT1A/1B/1D receptors antagonists with potent 5-HT reuptake inhibitory activity. Bioorg. Med. Chem. Lett. 2008, 5581-5585.

27. Wang, T.; Magnin, D.R.; Hamann, L.G. Palladium-catalyzed microwave-assisted amination of 1-bromonaphthalenes and 5- and 8-bromoquinolines. Org. Lett. 2003, 5, 897-900.

28. Childers, W.E.; Havran, L.M.; Asselin, M.; Bicksler, J.J.; Chong, D.C.; Grosu, G.T.; Shen. Z.; Abou-Gharbia, M.A.; Bach, A.C.; Harrison, B.L.; et al. The Synthesis and Biological Evaluation of Quinolyl-piperazinyl Piperidines as Potent Serotonin 5-HT1A Antagonists. J. Med. Chem. 2010, 53, 4066-4084.

29. Xie, Y.-X.; Pi, S.-F.; Wang, J.; Yin, D.-L.; Li, J.-H. 2-Aminopyrimidine-4,6-diol as an efficient ligand for solvent-free copper-catalyzed $\mathrm{N}$-arylations of imidazoles with aryl and heteroaryl halides. J. Org. Chem. 2006, 71, 8324-8327.

30. Klapars, A.; Antilla, J.; Huang, X.; Buchwald, S.L. A general and efficient copper catalyst for the amidation of aryl halides and the $N$ arylation of nitrogen heterocycles. J. Am. Chem. Soc. 2001, 123, 7727-7729.

31. Klapars, A.; Huang, X.; Buchwald, S.L. A general and efficient copper catalyst for the amidation of aryl halides. J. Am. Chem. Soc. 2002, 124, 7421-7428.

32. Xie, J.; Ning, Z.; Tian, H. A soluble 5-carbazolium-8-hydroxyquinoline Al(III) complex as a dipolar luminescent material. Tetrahedron Lett. 2005, 46, 8559-8562.

33. Wolf, C.; Lerebours, R. Use of highly active palladium-phosphinous acid catalysts in Stille, Heck, amination, and thiation reactions of chloroquinolines. J. Org. Chem. 2003, 68, 7077-7084. 
34. Melato, S.; Coghi, P.; Basilico, N.; Prosperi, D.; Monti, D. Novel 4-aminoquinolines through microwave-assisted SNAr reactions: A practical route to antimalarial agents. Eur. J. Org. Chem. 2007, 2007, 6118-6123.

35. Girault, S.; Grellier, P.; Berecibar, A.; Maes, L.; Lemiere, P.; Mouray, E.; Davioud-Charvet, E.; Sergheraert, C. Antiplasmodial activity and cytotoxicity of bis-, tris-, and tetraquinolines with linear or cyclic amino linkers. J. Med. Chem. 2001, 44, 1658-1665.

36. Musonda, C.C.; Whitlock, G.A.; Witty, M.J.; Brun, R.; Kaiser, M. Chloroquine-astemizole hybrids with potent in vitro and in vivo antiplasmodial activity. Bioorg. Med. Chem. Lett. 2009, 19, 481-484.

37. Averin, A.D.; Ulanovskaya, O.A.; Fedotenko, I.A.; Borisenko, A.A.; Serebryakova, M.V.; Beletskaya, I.P. Palladium-catalysed amination of 3,5-dihalopyridines-A convenient route to new polyazamacrocycles. Helv. Chim. Acta 2005, 88, 1983-2002.

38. Averin, A.D.; Ulanovskaya, O.A.; Pleshkova, N.A.; Borisenko, A.A.; Beletskaya, I.P. Pd-catalyzed amination of 2,6-dihalopyridines with polyamines. Collect. Czech. Chem. Commun. 2007, 72, 785-819.

39. Averin, A.D.; Uglov, A.N.; Buryak, A.K.; Bessmertnykh, A.G.; Guilard, R.; Beletskaya, I.P. Synthesis of polyazamacrocycles comprising 6,6'-diamino-2,2'-bipyridine moieties via Pd-catalyzed amination. Heterocycles 2010, 80, 957-975.

40. Grigorova, O.K.; Averin, A.D.; Abel, A.S.; Maloshitskaya, O.A.; Kovalev, V.V.; Savelev, E.N.; Orlinson, B.S.; Novakov, I.A.; Beletskaya, I.P. Arylation of adamantanamines: IV. Palladium-catalyzed arylation of amines of adamantane series with chloroquinoline isomers. Russ. J. Org. Chem. 2012, 48, 1391-1406.

41. Gopalan, B.; Thomas, A.; Shah, D.M. Preparation of adamantane derivatives as dipeptidyl peptidase IV inhibitors, and pharmaceutical compositions containing them. WO 2006090244 , 31 August 2006.

42. Novakov, I.A.; Kulev, I.A.; Radchenko, S.S.; Birznieks, K.A.; Boreko, E.I.; Vladyko, G.V.; Korobchenko, L.V. Synthesis and antiviral activity of hydrochlorides of alicyclic mono- and diamines. Pharm. Chem. J. 1987, 21, 454-458.

43. Averin, A.D.; Ranyuk, E.R.; Golub, S.L.; Buryak, A.K.; Savelyev, E.N.; Orlinson, B.S.; Novakov, I.A.; Beletskaya, I.P. Synthesis of a new family of adamantylpyridin-2-amines via Pd-catalyzed amination. Synthesis 2007, 2007, 2215-2221.

44. Ukai, T.; Kawazura, H.; Ishii, Y.; Bonnet, J.J.; Ibers, J.A. Chemistry of dibenzylideneacetonepalladium(0) complexes I. Novel tris(dibenzylideneacetone)-dipalladium(solvent) complexes and their reactions with quinines. J. Organomet. Chem. 1974, 65, 253-266.

Sample Availability: Not available.

(C) 2013 by the authors; licensee MDPI, Basel, Switzerland. This article is an open access article distributed under the terms and conditions of the Creative Commons Attribution license (http://creativecommons.org/licenses/by/3.0/). 